\title{
Novel Masters of Erythropoiesis: Hypoxia Inducible Factors and Recent Advances in Anemia of Renal Disease
}

\author{
Yalcin Solak $^{\mathrm{a}}$ Mustafa Cetiner $^{\mathrm{b}}$ Dimitrie Siriopol $^{\mathrm{c}}$ Kayhan Tarim $^{\mathrm{d}}$ \\ Baris Afsar ${ }^{\mathrm{e}}$ Adrian Covic $^{c}$ Mehmet Kanbay ${ }^{f}$ \\ ${ }^{a}$ Division of Nephrology, Department of Medicine, Sakarya University Training and Research Hospital, Sakarya, \\ ${ }^{b}$ Division of Hematology, Department of Medicine, Koc University School of Medicine, Istanbul, Turkey; \\ 'Nephrology Clinic, Dialysis and Renal Transplant Center, 'C.I. PARHON' University Hospital, and 'Grigore T. Popa' \\ University of Medicine, Iasi, Romania; 'Department of Medicine, Koc University School of Medicine, Istanbul, \\ e Division of Nephrology, Department of Nephrology, Konya Numune State Hospital, Konya, ${ }^{\mathrm{f} D i v i s i o n}$ of \\ Nephrology, Department of Medicine, Koc University School of Medicine, Istanbul, Turkey
}

\section{Key Words}

Aneamia $\cdot$ Inflammation Chronic kidney disease .

Hepcidin · Hypoxia inducible factors

\section{Abstract}

Anemia seen in patients with chronic kidney disease is a particular form of 'anemia of chronic disease'. Although multifactorial in origin, erythropoiesis-stimulating agents (ESAs) and adjuvant iron therapy represent the primary treatment for anemia in chronic kidney disease. Subsequent clinical observations revealed that these ESA hyporesponsive patients often had increased systemic inflammation as a consequence of their comorbidities. Use of high ESA doses to overcome this ESA hyporesponsiveness posed some concerns regarding associated adverse events of therapy and increased mortality in this special patient population. Recognizing the pivotal roles of hypoxia inducible factors (HIFs) in orchestrating elements of erythropoiesis opened new avenues in the management of renal anemia. Several phase 1 and 2 studies confirmed the results of early experimental studies supporting the beneficial role of augmenting HIFs for erythropoiesis. In this review, we describe the physiolog- ic functions of HIF in erythropoiesis with special emphasis on interactions with iron and hepcidin metabolism and inflammation.

c 2016 S. Karger AG, Basel

\section{Introduction}

Anemia seen in patients with chronic kidney disease is a particular form of 'anemia of chronic disease'. Although multifactorial in origin, the relative deficiency of serum erythropoietin (EPO) levels to the level of hemoglobin is considered as the salient pathophysiologic factor. Advent of recombinant human EPO as a therapeutic option heralded a new era in which numbers of red blood cell transfusions steeply decreased along with attendant increases in serum hemoglobin levels and increased quality of life [1]. However, a subset of patients treated with erythropoiesis stimulating agents (ESAs) remained anemic despite very high ESA doses utilized [2,3]. Subsequent clinical observations revealed that these ESA hyporesponsive patients often had increased systemic inflammation as a consequence of their comorbidities. The underpinnings

\section{KARGER}

E-Mail karger@karger.com

www.karger.com/bpu
(C) 2016 S. Karger AG, Basel

0253-5068/16/0422-0160\$39.50/0
Mehmet Kanbay, MD

Division of Nephrology, Department of Medicine

Koc University School of Medicine

Sariyer, TR-34010 Istanbul (Turkey)

E-Mail mkanbay@ku.edu.tr, drkanbay@yahoo.com 
Fig. 1. HIFs affect several distinct components of iron metabolism as well as EPO production in kidney. Note that HIFs do not directly affect hepcidin synthesis, HIFinduced reduction in serum hepcidin levels is believed to be through its effects through iron metabolism.

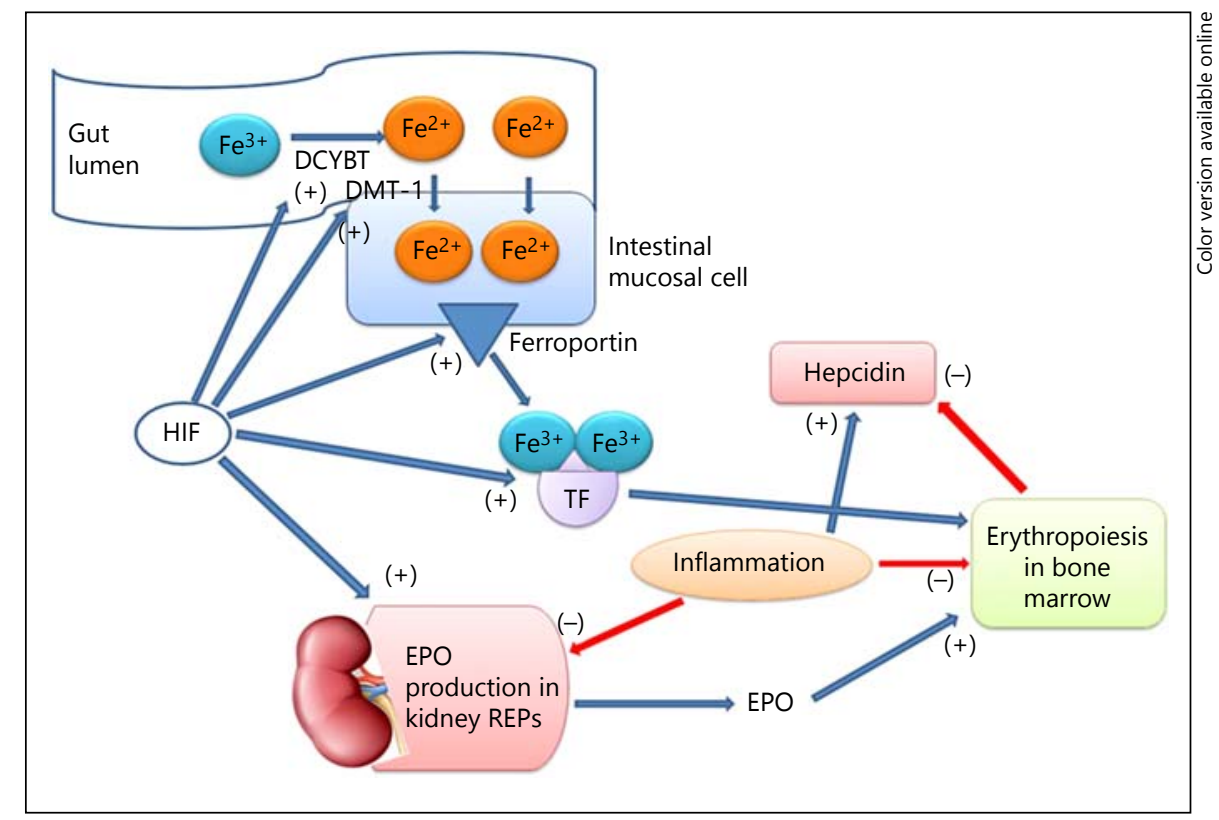

of this inflammation-anemia relationship were rendered clearer after discovery of hepcidin as the master regulator of iron metabolism. Use of high ESA doses to overcome this ESA hyporesponsiveness posed some concerns regarding associated adverse events of therapy and increased mortality in this special patient population [4].

Hypoxia inducible factors (HIFs) are heterodimeric proteins that regulate the physiologic response to hypoxia by altering gene expression with consequences of increased EPO production and improved uptake and utilization of iron, among many others. Recognizing the pivotal roles of HIFs in orchestrating elements of erythropoiesis opened new avenues in the management of renal anemia [5]. Several phase 1 and 2 studies confirmed the results of early experimental studies supporting the beneficial role of augmenting HIFs for erythropoiesis. As a more upstream location and playing the role of 'real master' in the regulation of erythropoiesis, HIFs were suggested as a likely way for a physiologic treatment of anemia without the untoward effects seen with ESA treatment [5]. On the other hand, this upstream location and role of master switch comes of course with justifiable concerns in terms of adverse events. In this review, we describe the physiologic functions of HIF in erythropoiesis with special emphasis on interactions with iron and hepcidin metabolism and inflammation. We will also review the most recent clinical data regarding HIF stabilizers, molecules that inhibit prolyl-4-hydroxilase domain (PHD) proteins, in the treatment of renal anemia.

\section{HIFs and Iron Metabolism}

HIFs affect nearly all aspects of iron metabolism related to erythropoiesis (fig. 1). HIF-2 enhances iron absorption from small bowel enterocytes and also augments iron export from duodenal cells. HIFs are also involved in the utilization of iron from treatment of senescent red blood cells via reticuloendothelial system macrophages. This way HIFs increase supply of iron from both absorption from duodenum and from the recycling of senescent red blood cells. This increased efflux of iron to the circulation is achieved via HIF mediated increased expression of ferroportin in duodenal enterocytes and macrophages [6]. HIF induces an increase in serum transferrin levels, the only transporter of iron in serum, which commensurate with an increased iron supply to circulation. In summary, HIFs oppose the actions of hepcidin in iron metabolism at several levels.

\section{HIFs and Hepcidin}

Hepcidin expression is increased by iron loading to avoid an excess of free toxic iron accumulation in the body, whereas it is decreased by iron deficiency, hypoxia and increased erythropoiesis to compensate iron supply for erythropoietic demand [7]. However, some controversy exists as to whether HIFs interact with hepatic hepcidin production in regulation of the iron metabolism. 
Nicolas et al. [8] demonstrated that hypoxia and anemia robustly decrease hepcidin gene expression. Turpentine induced inflammation increased liver hepcidin mRNA levels, whereas this effect was blunted in hepcidin deficient mice. Braliou et al. [9] also confirmed the importance of 2-oxoglutarate-dependent oxygenases (namely PHDs) in the hypoxic suppression of hepcidin. The authors applied hypoxia and inhibitors of 2-oxoglutarate-dependent oxygenases to cultured human hepatoma cells and observed the downregulation of hepcidin gene promoter activity as well as hepcidin m-RNA levels [9]. Interestingly, this effect was still operative even under interleukin-6 treatment. In contrast to the latter studies, Volke et al. [10] reported that hypoxia does not consistently suppress hepcidin expression in human hepatoma cells. On the other hand, the authors showed reproducible reduction in hepcidin mRNA levels with chemical HIF stabilizers independent of HIF- $1 \alpha$, HIF- $2 \alpha$, transferrin receptor 1 induction. Rather this suppression was in parallel to regulation of transferrin receptor 2 , one of the genes critical to hepcidin expression [10].

Later experimental work pointed to an indirect role of HIFs in regulation of hepcidin production. First, Mastrogiannaki et al. [11] demonstrated that HIF-2 is not involved in the downregulation of hepcidin in case of iron deficiency. However, HIF-2 overexpression in the double Vhlh/HIF-1a hepatocyte-specific knockout mice, indirectly downregulated hepcidin expression through increased EPO production. The findings of the latter study were confirmed by the study of Liu et al. [12], in which the authors used a genetic approach to separate HIF activation from EPO synthesis and demonstrated that HIF-mediated suppression of the hepcidin gene required EPO induction. Thus, it was assumed that hepcidin gene suppression was not due to direct effect of HIFs but rather secondary to erythropoiesis induced by EPO.

Although precise mechanisms by which HIFs decrease hepcidin levels are yet to be elucidated further, recent clinical trials also confirmed the earlier experimental data with respect to suppressive effects of HIFs on hepcidin $[13,14]$. In a recent open-label, randomized study performed in anemic patients incident to hemodialysis or peritoneal dialysis, the administration of roxadustat, an orally bioavailable HIF prolyl hydroxylase inhibitor, decreased the mean hepcidin levels at 4 weeks [13]. The reduction was most remarkable in hemodialysis patients who received no iron treatment at all. Reduction in hepcidin levels continued in patients who had received oral iron or received no iron, whereas hepcidin levels re- bounded in patients who received intravenous iron at the end of the 12-week treatment period. The authors speculated that this may be due to competing feedback loops by higher circulating iron levels provided by intravenous iron treatment [13]. Another placebo controlled clinical trial showed that 4 weeks treatment of roxadustat significantly reduced serum hepcidin levels compared with baseline pretreatment values in patients with pre-dialysis chronic kidney disease [14]. But in another phase 2a study by Holdstock et al. [15] GSK1278863 (a HIF prolyl hydroxylase inhibitor) reduced serum hepcidin levels significantly in pre-dialysis CKD patients compared with placebo in $5 \mathrm{mg}$ dose group only. Interestingly, meaningful serum hemoglobin increase was only achieved in that arm. On the other hand, in the parallel study conducted on hemodialysis patients, in which patients on stable ESA doses served as control group, hepcidin levels were not changed with either ESA treatment or 5 mg GSK1278863. In the latter study, in lower dose arms of GSK1278863, mean hemoglobin levels were not maintained and serum hepcidin levels in fact increased from the baseline. These observations also imply that HIF prolyl hydroxylase inhibitors seem to repress hepcidin levels through increase in hemoglobin levels.

\section{REPs and Their Characteristics}

Beginning with the discovery that kidneys are the major source of circulating EPO, search for the original cells producing EPO and quest to characterize them have continued to date. Several researchers attempted to identify these cells with variable methods but it has to be waited until development of transgenic methods. In situ hybridization and immunohistochemical methods in renal tissue sections did not yield clearcut results due to the inherent limitations of sensitivity and specificity of these methods [16]. Transgenic mouse models pointed to the possibility that renal fibroblasts may be at the top of the list of potential candidate cells for EPO production [17]. Subsequent experimental work by Yamazaki et al. [18] utilizing bacterial artificial chromosomes transgenic mice, green fluorescent protein reporter knock-in mice and a genetically modified mouse line revealed that renal EPO producing cells (REPs) were fibroblast-like cells residing around peritubular capillaries in the cortex and outer medulla of the kidney. These cells were expressing neural markers such as neurofilament light peptide, nerve growth factor receptor. Subsequent studies elicited some interesting findings regarding REPs. First, these cells were not abundant under steady 
Fig. 2. REPs are recently characterized cells principally responsible for EPO synthesis. Under persistent renal injury, these cells transform into myofibroblast cells which have the capacity to produce pro-fibrotic cytokines and extracellular matrix. In addition, when REPs turned into myofibroblast, they lose their ability to produce EPO. Notably, and fortunately, recent research showed that REPs may go back their original state and regain their EPO synthesis capability once renal injury halts.

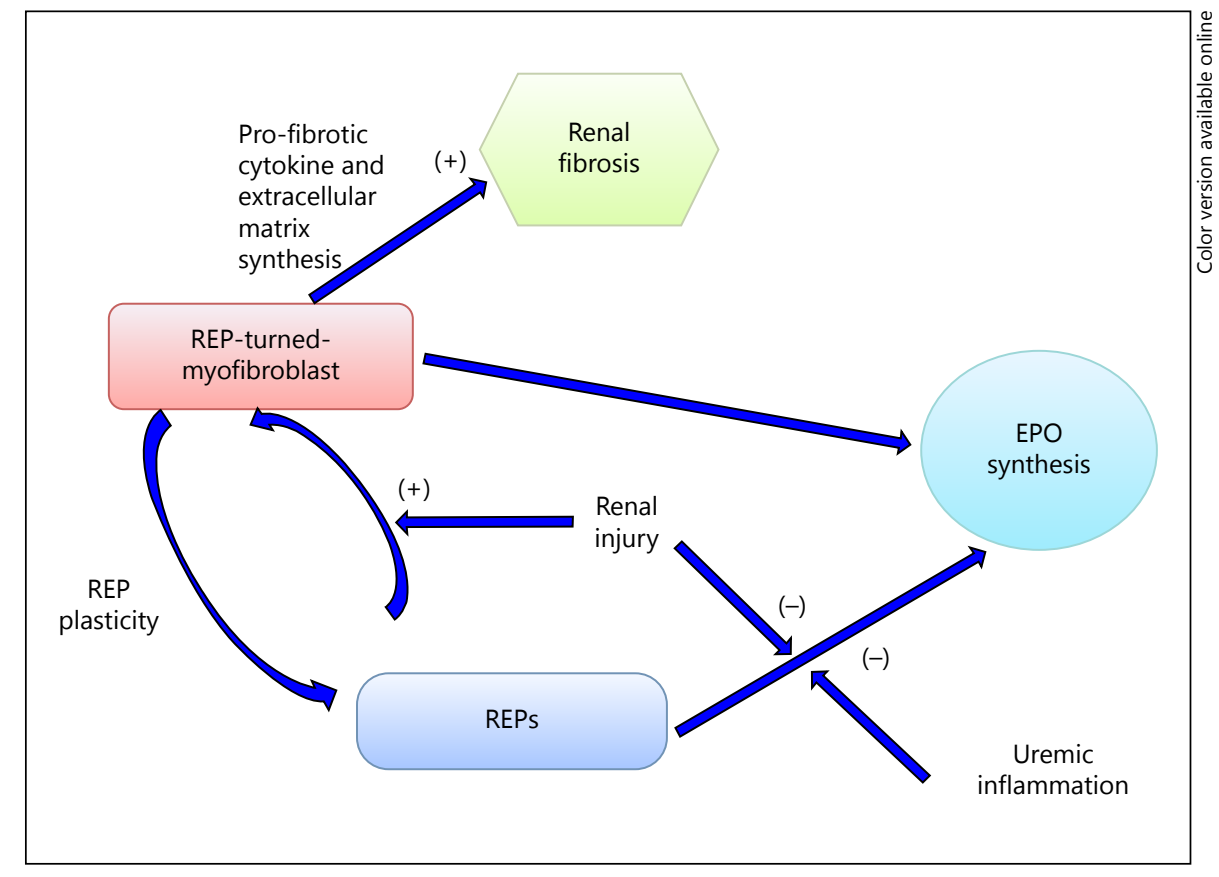

state conditions. Chronic anemia, but not acute anemia, was a prime stimulus for EPO gene expression in REPs [18]. Hypoxia is the main regulatory factor for EPO production in REPs. Hypoxia in the kidney, but not in other organs, is critical for EPO gene expression and resultant EPO production from REPs. This finding is also consistent with the observation that REPs reside in the kidney around peritubular capillaries where they can sense level of hypoxia through HIFs [18]. Hypoxia also causes spread of REPS from their original sites, juxtamedullary region, to encompass the whole cortex. Second, though having the capacity of EPO production, most of the REPs (90\% of total REP pool) remain in the resting state even under severe hypoxic insult. Renal EPO production is regulated with an 'ON-OFF' fashion; while EPO producing cells are active $(\mathrm{ON})$ they express EPO genes, on the other hand if they are not actively producing EPO they do not express EPO genes and are in an OFF state $[16,19]$. Third, hypoxia modulates REPs through HIFs [20].

Some unintended findings were discovered while trying to better characterize REPs with respect to EPO production. Accruing data have shown that under fibrotic insults, REPs transform into avid myofibroblasts with a capability to secrete extracellular matrix and inflammatory cytokines [19]. Subsequent experimental work showed that REP-turned myofibroblasts are crucial for initiation and propagation of renal fibrosis (fig. 2). Timehonored clinical observations that patients with primary tubulointerstitial disease have earlier and more severe anemia compared with their glomerulopathic counterparts and are in agreement with recent discoveries on the residence and functioning of REPs and on injury stimulated trans-differentiating of REPs to myofibroblasts. In addition to their new capabilities with respect to fibrogenesis and cytokine secretion, myofibroblasts also lose their inherent capability of producing EPO. Equally exciting was the finding that REPs regain their inherent characteristics including EPO production once the injurious stimulation disappears [19]. This so-called plasticity is of considerable importance both for the slowing or stopping of renal fibrogenesis, which is the final common pathway of nearly all renal disorders and for the treatment of renal anemia characterized by EPO depletion.

\section{HIFs as Master Regulator of EPO Synthesis}

Discovery of HIFs filled the gap in our understanding on how anemia and/or hypoxia may lead to augmentation of erythropoiesis. HIFs are heterodimeric proteins consisting of oxygen sensitive $\alpha$ and constitutive $\beta$ subunits. HIFs coordinate cellular responses to hypoxia, and induction of erythropoiesis is one of these responses. Mice with knock-out HIF had anemia, whereas activating mutations in the HIF pathway are associated with the development of polycythemia $[21,22]$. 
Three HIF- $\alpha$ subunits have been described to date: HIF-1 $\alpha$, HIF- $2 \alpha$ and HIF-3 $\alpha$. However, systemic knockout studies have revealed HIF- $2 \alpha$ as the most potent HIF in the regulation of renal EPO production [23]. HIF-2a is also the major HIF involved in iron metabolism [24]. Under normoxemic conditions, HIF- $\alpha$ subunits are hydroxylated by 2-oxoglutarate-dependant oxygenase - PHD. Proline-hydroxylated HIF- $2 \alpha$ is ubiquitylated by the von Hippel-Lindau ubiquitin ligase complex and then directed rapidly for proteosomal degradation [25]. Once hypoxia is developed, hydroxylation by PHDs is inhibited and HIFs escape degradation and accumulate. Pan et al. [26] demonstrated that all 3 PHDs are available in renal EPO-producing cells isolated by fluorescence-activated cell sorting. PHD2 is the main enzyme regulating HIF activity under normal oxygen tension. It has been shown that inherited mutations in PHD2 that inhibit enzymatic activity are associated with the development of erythrocytosis [27].

In addition to kidney, hypoxia-regulated EPO transcription also occurs in the liver. Similar to the case in the kidney, liver-inducibility element in EPO gene is required for induction by hypoxia and resultant EPO production [28]. Although negligible contribution to total EPO synthesis in adults, liver is the major source of extra-renal EPO production and can generate $25-50 \%$ of circulating EPO under moderate to severe hypoxic conditions [29]. Kapitsinou et al. [30] utilized an elaborate experimental model in that Cre-loxP recombination performed to ablate HIF-2 $\alpha$ in the kidney, whereas HIF-2-mediated hypoxia responses in the liver and other EPO-producing tissues remained intact to assess the role of liver in the absence of EPO production by kidney. The authors demonstrated that in the absence of renal HIF-2, hepatic HIF-2 takes over as the major producer of EPO. In addition, exposure to hypoxia and HIF stabilizer administration led to a 15- and 1,500-fold increase in hepatic EPO mRNA levels, respectively.

\section{HIFs and Inflammation}

Inflammation is of paramount importance for the development of anemia of chronic disease. Chronic kidney disease is also considered as a state of increased inflammation [31]. Increased inflammation can affect several components of erythropoiesis including bone marrow erythroblastic development, iron metabolism and inhibition of EPO production [3].

Certain proinflammatory cytokines may suppress erythroid progenitor cell proliferation in the bone marrow [32]. Inhibition of EPO production by increased inflam- mation also suppresses erythropoiesis. Faquin et al. [33] showed that IL- $1 \alpha$, IL- $1 \beta$ and TNF- $\alpha$ led to a dose-dependent inhibition of hypoxia-induced EPO production in the Hep3b cell line.

The exact mechanism by which inflammation suppresses EPO production is largely unknown. However, very recent experimental data imply that HIFs may be the missing link in this relationship. Souma et al. [34] showed that PHD deletion in REPs attenuates the lipopolysaccharide-induced EPO gene suppression, and the inflammatory milieu may accelerate HIF degradation. This observation is very important in EPO-resistant patients with increased inflammation, because pharmacologic HIF stabilization may counterbalance effects of inflammation on EPO production.

\section{HIF Stabilizers as Anti-Anemic Agents in Clinical Practice}

Experimental data describing the central regulator role of HIFs in erythropoiesis and iron metabolism led to the development of HIF stabilizers as a novel way of treating EPO-deficient anemia.

Several recent experimental studies demonstrated that HIF stabilization through genetic deletion of PHDs may allow REPs to regain their EPO-producing capacity [19, 34]. HIF stabilizers are competitive inhibitors of PHDs. By inhibiting PHDs, these drugs allow endogenous HIF to accumulate and simulate the naturally occurring hypoxia, through which they augment EPO gene expression and EPO production.

There are several HIF stabilizers at various stages of development. Hydroxyisoquinoline-based compounds, FG-2216 and FG-4592 (roxadustat), were the first HIF stabilizers. Further phase studies with FG-2216 were suspended because of a fatal case of hepatitis. Roxadustat has been evaluated both in dialysis-dependent and pre-dialysis chronic kidney disease patients. In a recent phase $2 \mathrm{a}$ study by Besarab et al. [14], 116 patients with pre-dialysis $\mathrm{CKD}$ were randomized to receive roxadustat 2 or 3 times weekly or placebo. Hemoglobin increased in a dose-dependent manner with roxadustat-treated patients. Rate of hemoglobin responders ranged from $30 \%$ in the $0.7 \mathrm{mg} /$ $\mathrm{kg}$ twice weekly group to $100 \%$ in the $2.0 \mathrm{mg} / \mathrm{kg}$ twice weekly. In addition, roxadustat moderately increased endogenous EPO and reduced hepcidin levels. In the placebo group, endogenous EPO level did not show any change, whereas in patients in the $1 \mathrm{mg} / \mathrm{kg}$ twice weekly roxadustat group, endogenous EPO level began to rise $4 \mathrm{~h}$ 
after application of the drug, peaked at approximately 10th hour and returned to baseline within $48 \mathrm{~h}$. Median endogenous EPO level was 113 IU/l. Adverse event rates were comparable in both groups. In another open-label, randomized study [13] about incident anemic hemodialysis or peritoneal dialysis, patients were treated with roxadustat for 12 weeks. Roxadustat was administered orally thrice weekly for 12 weeks. Dose escalation based on evaluation of hemoglobin value every 4 weeks to a maximum of $2.5 \mathrm{mg} / \mathrm{kg}$ was performed. Twenty-four hemodialysis patients did not receive iron replacement in any form whereas 12 hemodialysis and 12 peritoneal dialysis patients received oral iron and 12 hemodialysis patients received intravenous iron replacement during treatment phase. Roxadustat increased mean hemoglobin $>2.0 \mathrm{~g} / \mathrm{dl}$ regardless of baseline iron status, C-reactive protein level, iron regimen or dialysis modality. There was no difference between the patients who received oral or parenteral iron in terms of hemoglobin response. Serum hepcidin levels significantly decreased with roxadustat treatment. Notably, roxadustat was effective in the majority of patients irrespective of baseline iron status and level of inflammation.

Holdstock et al. [15] conducted 2 parallel group phase 2a studies in pre-dialysis CKD and hemodialysis patients. GSK1278863 dose groups were $0.5,2$ or $5 \mathrm{mg}$. Placebo group and ESA-treated patients served as placebo groups in pre-dialysis CKD and hemodialysis groups, respectively. GSK1278863 produced dose-dependent increase on hemoglobin in the pre-dialysis CKD patients with the highest dose resulting in a mean increase of $1 \mathrm{~g} / \mathrm{dl}$ at fourth week of treatment. In hemodialysis patients, only treatment with $5 \mathrm{mg}$ GSK1278863 maintained mean hemoglobin concentrations after the switch from EPO treatment, but not other dose groups. Endogenous EPO levels increased with GSK1278863 treatment but levels were much less compared with those in the ESA group. Notably treatment with GSK1278863 was not accompanied by increases in vascular endothelial growth factor (VEGF) levels.

Some other HIF stabilizers including AKB-6548 (Aklebia Therapeutics), GSK1278863 (GlaxoSmithKline) and Molidustat (BAY 85-3934, Bayer Pharma) are in different phases of development, also showing a significant increase in hemoglobin levels in different CKD populations [5].

Some differences as compared to ESAs appear after the results of these preliminary studies. First, HIF stabilizers have the advantage of being administered orally. Second, HIF stabilizers increase endogenous EPO levels more closely resembling physiologic EPO production. Third, HIF stabilizers are effective irrespective of inflammation and level of iron repletion in anemic patients with CKD. Lastly, these short-term studies did not reveal increased adverse event rate compared to placebo $[13,14]$.

\section{Concerns with HIF Stabilizers}

No drug is without adverse effects and this is also true for HIF stabilizers. Although localized much distal in the pathway of erythropoiesis, ESAs are associated with some adverse effects. HIFs reside more proximal and interacts with a number of known (and certainly yet unknown) biological pathways. Thus, we should expect some untoward effects in some other organ systems when we simulate a pathologic state, namely hypoxia, to exploit for increasing the erythropoiesis.

HIFs are involved not only in erythropoiesis but also in a number of cellular responses including mitochondrial metabolism, cellular growth and differentiation, angiogenesis and anaerobic metabolism [35]. HIFs regulate numerous transcriptional responses and more than 500 HIF binding sites have been identified so far [36]. HIFs have been shown to affect vascular tone and blood pressure. In experimental models of chronic continuous hypoxia induced pulmonary hypertension, compared with wild type mice, a blunted response was observed in animals with HIF- $1 \alpha+/-$ and HIF- $2 \alpha+/-[37,38]$. In addition, it was shown that HIFs are associated with ocular neovascularization and systemic hypertension of sleep apnea $[39,40]$.

Accruing evidence has suggested that the balance between HIF- $1 \alpha$ and HIF- $2 \alpha$ is of crucial importance for the maintenance of systemic arterial pressure [41]. Transcription of HIF-1a leads to an increased expression of NO synthase 2 and consequently increased NO production whereas transcription of HIF- $2 \alpha$ indirectly reduces NO production $[42,43]$. There also appears a negative feedback control loop between HIF-1 $\alpha$ and NO. In summary, HIF-2 $\alpha$ inhibitors impair the balance of HIFs favoring HIF- $1 \alpha$ and theoretically are expected to decrease systemic blood pressure because of HIF-1 $\alpha$-induced NO release in the vasculature. However, there are scant data in clinical studies to support this. Another effect of PHD inhibitors on blood pressure may be their more physiologic effect on endogenous EPO levels and thereby preventing hypertensive effects of ESAs. Some experimental studies confirmed these theoretical assumptions [44]. Data regarding the blood pressure effects of HIF-PHD 
inhibitors are limited and conflicting; one phase $2 \mathrm{a}$ dose escalation study with AKB-6548 revealed small reductions in blood pressure whereas no significant change was observed in another phase 2a study with the same drug [41].

In many human cancers, HIF- $1 \alpha$ and HIF- $2 \alpha$ overexpression was found and increased HIF- $1 \alpha$ or HIF- $2 \alpha$ levels in the primary tumor were associated with increased mortality in solid tumors and hematologic malignancies. HIFs, particularly HIF-1 $\alpha$, play different roles in cancer pathogenesis including cell survival, autocrine signaling, metabolic reprogramming, neovascularization, immune evasion and metastasis [45]. Carroll and Ashcroft [46] showed contrasting effects of HIF- $1 \alpha$ and HIF- $2 \alpha$ in the regulation of HIF target gene expression in renal carcinoma cells that have lost VHL protein function and in response to IGF-I and hypoxia. The authors showed that HIF-1 $\alpha$, but not HIF-2 $\alpha$, primarily regulates VEGF expression in response to hypoxia and IGF-I. Several clinical studies also confirmed these results in which HIF-2a stabilizers did not increase serum VEGF levels [15]. But this does not necessarily mean that tissue level of VEGF expression is not affected by HIF stabilizers. This study also found that HIF- $2 \alpha$ knockdown enhanced HIF-1 $\alpha$ protein levels and VEGF expression hence implying that manipulation of one HIF molecule may affect somehow the others with possible biologic consequences. Considerably more research has implicated the role of HIF- $1 \alpha$ in the pathogenesis of cancer and many potential drugs interfering with this pathway are under development currently [47].

On the other hand, hypoxia-induced increase in HIFs have been shown to be protective against a number of disorders including, but not limited to, myocardial ischemia due to coronary artery disease, limb ischemia due to peripheral arterial disease, pressure-overload heart failure, wound healing and chronic rejection of organ transplants [45]. However, GSK1278863 failed to improve measures of performance in subjects with claudicationlimited peripheral artery disease in a multicenter ran- domized controlled study [48]. Since cardiovascular disease is the leading cause of death in patients with CKD, it is warranted for clinical trials to look for potential effects of HIF stabilizers on cardiovascular outcomes in treated patients.

\section{Conclusion}

HIFs were recognized as the master regulators of hypoxia-driven erythropoiesis. They also regulate iron metabolism and interacts with hepcidin. Recent characterization of EPO-producing cells showed that REPs transform into myofibroblasts and promote fibrosis in injured kidneys and they can transform back into their native state with augmentation of HIF levels. Pharmacologic manipulation of HIF pathway by means of HIF stabilizers opened new avenues in anemia treatment since the discovery of ESAs. Oral route of administration, efficacy even in iron depleted and increased inflammatory states, more physiologic simulation of endogenous EPO secretion and putative beneficial effects on renal fibrosis seem the main advantages of HIF stabilizers. Although safe in short-term clinical trials, long-term adverse events should be closely scrutinized.

\section{Disclosure Statement}

Authors have no conflict of interest.

\section{Author Contributions}

All authors approved the final version of manuscript.

\section{Financial Support}

None.

\section{References}

1 Finkelstein FO, Story K, Firanek C, Mendelssohn D, Barre P, Takano T, et al: Health-related quality of life and hemoglobin levels in chronic kidney disease patients. Clin J Am Soc Nephrol 2009;4:33-38.

2 Kanbay M, Perazella MA, Kasapoglu B, Koroglu M, Covic A: Erythropoiesis stimulatory agent-resistant anemia in dialysis patients: review of causes and management. Blood Purif 2010;29:1-12.
3 Yilmaz MI, Solak Y, Covic A, Goldsmith D, Kanbay M: Renal anemia of inflammation: the name is self-explanatory. Blood Purif 2011;32:220-225.

4 Zhang Y, Thamer M, Kaufman JS, Cotter DJ, Hernan MA: High doses of epoetin do not lower mortality and cardiovascular risk among elderly hemodialysis patients with diabetes. Kidney Int 2011;80:663-669.

\footnotetext{
5 Bonomini M, Del Vecchio L, Sirolli V, Locatelli F: New treatment approaches for the anemia of CKD. Am J Kidney Dis 2016;67: 133-142.

6 Taylor M, Qu A, Anderson ER, Matsubara T, Martin A, Gonzalez FJ, et al: Hypoxia-inducible factor- $2 \alpha$ mediates the adaptive increase of intestinal ferroportin during iron deficiency in mice. Gastroenterology 2011;140:2044-2055.
} 
7 Nemeth E, Ganz T: The role of hepcidin in iron metabolism. Acta Haematol 2009;122: 78-86.

8 Nicolas G, Chauvet C, Viatte L, Danan JL, Bigard X, Devaux I, et al: The gene encoding the iron regulatory peptide hepcidin is regulated by anemia, hypoxia, and inflammation. J Clin Invest 2002;110:1037-1044.

9 Braliou GG, Verga Falzacappa MV, Chachami G, Casanovas G, Muckenthaler MU, Simos G: 2-Oxoglutarate-dependent oxygenases control hepcidin gene expression. J Hepatol 2008;48:801-810.

10 Volke M, Gale DP, Maegdefrau U, Schley G, Klanke B, Bosserhoff AK, et al: Evidence for a lack of a direct transcriptional suppression of the iron regulatory peptide hepcidin by hypoxia-inducible factors. PLoS One 2009;4:e7875.

11 Mastrogiannaki M, Matak P, Mathieu JR, Delga S, Mayeux P, Vaulont S, et al: Hepatic hypoxia-inducible factor-2 down-regulates hepcidin expression in mice through an erythropoietin-mediated increase in erythropoiesis. Haematologica 2012;97:827-834.

12 Liu Q, Davidoff O, Niss K, Haase VH: Hypoxia-inducible factor regulates hepcidin via erythropoietin-induced erythropoiesis. J Clin Invest 2012;122:4635-4644.

13 Besarab A, Chernyavskaya E, Motylev I, Shutov E, Kumbar LM, Gurevich K, et al: Roxadustat (FG-4592): correction of anemia in incident dialysis patients. J Am Soc Nephrol 2016;27:1225-1233.

14 Besarab A, Provenzano R, Hertel J, Zabaneh R, Klaus SJ, Lee T, et al: Randomized placebocontrolled dose-ranging and pharmacodynamics study of roxadustat (FG-4592) to treat anemia in nondialysis-dependent chronic kidney disease (NDD-CKD) patients. Nephrol Dial Transplant 2015;30:1665-1673.

15 Holdstock L, Meadowcroft AM, Maier R, Johnson BM, Jones D, Rastogi A, et al: Fourweek studies of oral hypoxia-inducible factorprolyl hydroxylase inhibitor GSK1278863 for treatment of anemia. J Am Soc Nephrol 2016; 27:1234-1244.

16 Souma T, Suzuki N, Yamamoto M: Renal erythropoietin-producing cells in health and disease. Front Physiol 2015;6:167.

17 Maxwell PH, Osmond MK, Pugh CW, Heryet A, Nicholls LG, Tan CC, et al: Identification of the renal erythropoietin-producing cells using transgenic mice. Kidney Int 1993;44: 1149-1162.

18 Yamazaki S, Souma T, Hirano I, Pan X, Minegishi N, Suzuki N, et al: A mouse model of adult-onset anaemia due to erythropoietin deficiency. Nat Commun 2013;4:1950.

19 Souma T, Yamazaki S, Moriguchi T, Suzuki N, Hirano I, Pan X, et al: Plasticity of renal erythropoietin-producing cells governs fibrosis. J Am Soc Nephrol 2013;24:1599-1616.

20 Haase VH: Hypoxic regulation of erythropoiesis and iron metabolism. Am J Physiol Renal Physiol 2010;299:F1-F13.

21 Yoon D, Pastore YD, Divoky V, Liu E, Mlodnicka AE, Rainey K, et al: Hypoxia-inducible factor-1 deficiency results in dysregulated erythropoiesis signaling and iron homeostasis in mouse development. J Biol Chem 2006; 281:25703-25711.

22 Lee FS, Percy MJ: The HIF pathway and erythrocytosis. Annu Rev Pathol 2011;6:165192.

23 Gruber M, Hu CJ, Johnson RS, Brown EJ, Keith B, Simon MC: Acute postnatal ablation of Hif-2alpha results in anemia. Proc Natl Acad Sci U S A 2007;104:2301-2306.

24 Mastrogiannaki M, Matak P, Keith B, Simon MC, Vaulont S, Peyssonnaux C: HIF-2alpha, but not HIF-1alpha, promotes iron absorption in mice. J Clin Invest 2009;119:11591166.

25 Koury MJ, Haase VH: Anaemia in kidney disease: harnessing hypoxia responses for therapy. Nat Rev Nephrol 2015;11:394-410.

26 Pan X, Suzuki N, Hirano I, Yamazaki S, Minegishi N, Yamamoto M: Isolation and characterization of renal erythropoietin-producing cells from genetically produced anemia mice. PLoS One 2011;6:e25839.

27 Percy MJ, Zhao Q, Flores A, Harrison C, Lappin TR, Maxwell PH, et al: A family with erythrocytosis establishes a role for prolyl hydroxylase domain protein 2 in oxygen homeostasis. Proc Natl Acad Sci U S A 2006;103: 654-659.

28 Suzuki N, Obara N, Pan X, Watanabe M, Jishage K, Minegishi N, et al: Specific contribution of the erythropoietin gene $3^{\prime}$ enhancer to hepatic erythropoiesis after late embryonic stages. Mol Cell Biol 2011;31:3896-3905.

29 Tan CC, Eckardt KU, Firth JD, Ratcliffe PJ: Feedback modulation of renal and hepatic erythropoietin mRNA in response to graded anemia and hypoxia. Am J Physiol 1992;263(3 pt 2):F474-F481.

30 Kapitsinou PP, Liu Q, Unger TL, Rha J, Davidoff O, Keith B, et al: Hepatic HIF-2 regulates erythropoietic responses to hypoxia in renal anemia. Blood 2010;116:3039-3048.

31 Akchurin OM, Kaskel F: Update on inflammation in chronic kidney disease. Blood Purif 2015;39:84-92.

32 Allen DA, Breen C, Yaqoob MM, Macdougall IC: Inhibition of CFU-E colony formation in uremic patients with inflammatory disease: role of IFN-gamma and TNF-alpha. J Investig Med 1999;47:204-211.

33 Faquin WC, Schneider TJ, Goldberg MA: Effect of inflammatory cytokines on hypoxiainduced erythropoietin production. Blood 1992;79:1987-1994.

34 Souma T, Nezu M, Nakano D, Yamazaki S, Hirano I, Sekine H, et al: Erythropoietin synthesis in renal myofibroblasts is restored by activation of hypoxia signaling. J Am Soc Nephrol 2016;27:428-438.

35 Semenza GL: HIF-1 and mechanisms of hypoxia sensing. Curr Opin Cell Biol 2001;13: 167-171.

36 Schodel J, Mole DR, Ratcliffe PJ: Pan-genomic binding of hypoxia-inducible transcription factors. Biol Chem 2013;394:507-517.
37 Yu AY, Shimoda LA, Iyer NV, Huso DL, Sun $\mathrm{X}, \mathrm{McW}$ illiams R, et al: Impaired physiological responses to chronic hypoxia in mice partially deficient for hypoxia-inducible factor 1alpha. J Clin Invest 1999;103:691-696.

38 Brusselmans K, Compernolle V, Tjwa M, Wiesener MS, Maxwell PH, Collen D, et al: Heterozygous deficiency of hypoxia-inducible factor-2alpha protects mice against pulmonary hypertension and right ventricular dysfunction during prolonged hypoxia. J Clin Invest 2003;111:1519-1527.

39 Yuan G, Nanduri J, Khan S, Semenza GL, Prabhakar NR: Induction of HIF-1alpha expression by intermittent hypoxia: involvement of $\mathrm{NADPH}$ oxidase, Ca2+ signaling, prolyl hydroxylases, and mTOR. J Cell Physiol 2008;217:674-685.

40 Ozaki H, Yu AY, Della N, Ozaki K, Luna JD, Yamada $\mathrm{H}$, et al: Hypoxia inducible factor1alpha is increased in ischemic retina: temporal and spatial correlation with VEGF expression. Invest Ophthalmol Vis Sci 1999;40:182189.

41 Yousaf F, Spinowitz B: Hypoxia-Inducible factor stabilizers: a new avenue for reducing BP while helping hemoglobin? Curr Hypertens Rep 2016;18:23.

42 Takeda N, O’Dea EL, Doedens A, Kim JW, Weidemann A, Stockmann C, et al: Differential activation and antagonistic function of HIF- $\{$ alpha\} isoforms in macrophages are essential for NO homeostasis. Genes Dev 2010; 24:491-501.

43 Nagai M, Terao S, Vital SA, Rodrigues SF, Yilmaz G, Granger DN: Role of blood cellassociated angiotensin II type 1 receptors in the cerebral microvascular response to ischemic stroke during angiotensin-induced hypertension. Exp Transl Stroke Med 2011;3: 15.

44 Flamme I, Oehme F, Ellinghaus P, Jeske M, Keldenich J, Thuss U: Mimicking hypoxia to treat anemia: HIF-stabilizer BAY 85-3934 (Molidustat) stimulates erythropoietin production without hypertensive effects. PLoS One 2014;9:e111838.

45 Semenza GL: Oxygen sensing, hypoxia-inducible factors, and disease pathophysiology. Annu Rev Pathol 2014;9:47-71.

46 Carroll VA, Ashcroft M: Role of hypoxia-inducible factor (HIF)-1alpha versus HIF-2alpha in the regulation of HIF target genes in response to hypoxia, insulin-like growth factor-I, or loss of von Hippel-Lindau function: implications for targeting the HIF pathway. Cancer Res 2006;66:6264-6270.

47 Masoud GN, Li W: HIF-1 1 a pathway: role, regulation and intervention for cancer therapy. Acta Pharm Sin B 2015;5:378-389.

48 Olson E, Demopoulos L, Haws TF, Hu E, Fang Z, Mahar KM, et al: Short-term treatment with a novel HIF-prolyl hydroxylase inhibitor (GSK1278863) failed to improve measures of performance in subjects with claudication-limited peripheral artery disease. Vasc Med 2014;19:473-482. 